\title{
SUPPORTING INFORMAITON
}

\section{Distinguishing Condensed-Phase Cracking Products with the Same Mass-to-Charge Ratio Using A Triple Quadrupole Mass Spectrometer in Product Scan Mode}

\author{
Andrew F. DeBlase, ${ }^{\mathrm{a}, \mathrm{b}^{*}}$ Christopher R. Bruening, ${ }^{\mathrm{b}, \mathrm{c}}$ William K. Lewis, ${ }^{\mathrm{b}}$ and \\ Christopher E. Bunker ${ }^{b}$ \\ ${ }^{a}$ Spectral Energies, LLC, Beavercreek, OH 45431, USA \\ ${ }^{\mathrm{b}}$ Air Force Research Laboratory, Aerospace Systems Directorate, Wright-Patterson Air Force \\ Base, Dayton, $\mathrm{OH}$ 45433, USA \\ ${ }^{c}$ University of Dayton Research Institute, Dayton, OH 45469, USA
}

\section{Description of the Experiment:}

The apparatus for in situ mass spectrometry samples the neat fluid at high temperature and pressure via a supersonic expansion from a stainless steel nozzle into the gas phase. After skimming the expansion jet, a molecular beam is formed, which is probed via electron-impact (EI) ionization followed by MS using a quadrupole mass filter. This technique allows us to obtain a "snapshot" of the condensed phase chemistry because further bimolecular chemistry becomes unlikely following expansion and skimming.

The instrument was configured for product scan mode. According to this method, target ions are isolated according to their $\mathrm{m} / \mathrm{z}$ in $\mathrm{Q} 1$ and subjected to collisions in $\mathrm{q} 2$ at a pressure of $4.4 \times 10^{-4}$ Torr of argon collision gas and a beam energy of $8 \mathrm{eV}$. Note that this pressure was measured using a cold cathode gauge connected to $\mathrm{q} 2$ via tubing (provided by Extrel) and the beam energy was set using the potential difference between the repeller and extractor plates in the ion source region. Because the mean free path at these conditions is approximately $20 \mathrm{~cm}$, we expect that the CID process occurs in the regime of few collisions, which impart bond-breaking energies because of the harsh transfer conditions into q2. The product ions are analyzed by scanning Q3.

\footnotetext{
${ }^{*}$ Corresponding author email: andrew.deblase@spectralenergies.com
} 


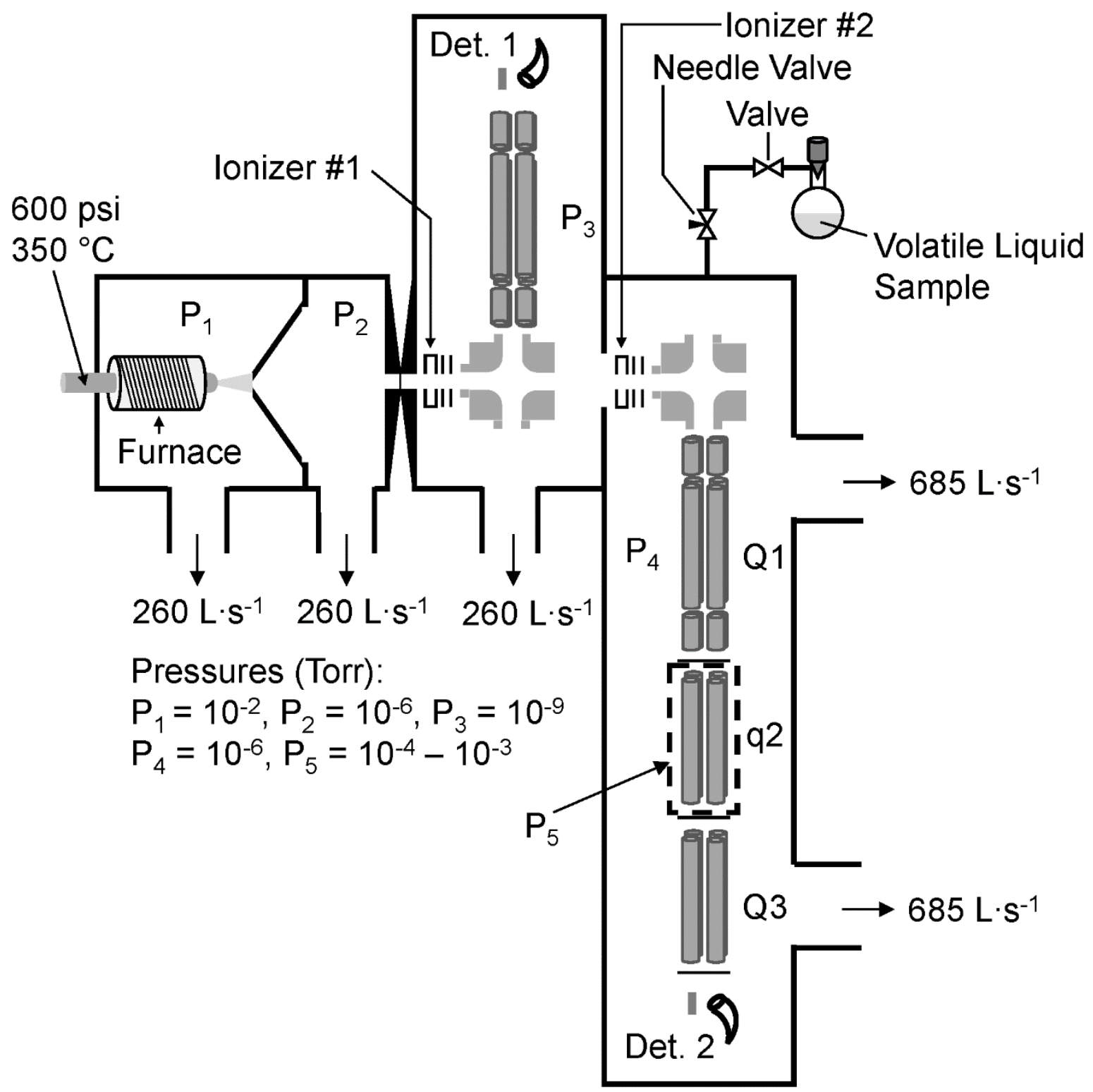

Figure S.1. (adapted from DeBlase et al. Energy Fuels 2019, 33, 10861) Molecular beam instrument for probing supercritical pyrolysis using both single quadrupole and triple quadrupole mass spectrometers. The molecular beam is ionized by two separated EI ionizers $(10 \mathrm{eV})$ and ions are turned into each mass spectrometer using an ion bender. Pressures and pumping speeds in each region of differential pumping are shown. The vapor leak-in source that was used to sample the head space of the volatile liquid compounds in this study is also shown, which is attached to the triple quadrupole section of the instrument (near Ionizer \#2). 